\title{
RADIO GALAXY FEEDBACK IN X-RAY-SELECTED GROUPS FROM COSMOS: THE EFFECT ON THE INTRACLUSTER MEDIUM
}

\author{
S. Giodini ${ }^{1}$, V. Smolčíc ${ }^{2}$, A. Finoguenov ${ }^{1,3}$, H. Boehringer ${ }^{1}$, L. Bîrzan ${ }^{4}$, G. Zamorani ${ }^{5}$, A. Oklopčíc ${ }^{6}$, D. Pierini ${ }^{1}$, \\ G. W. Pratt ${ }^{7}$, E. Schinnerer ${ }^{8}$, R. Massey ${ }^{2}$, A. M. Koekemoer ${ }^{9}$, M. Salvato ${ }^{10}$, D. B. Sanders ${ }^{11}$, J. S. Kartaltepe ${ }^{11}$, \\ AND D. THOMPSON ${ }^{12}$ \\ ${ }^{1}$ Max Planck Institut für Extraterrestrische Physik, Giessenbachstrasse, Garching bei München D-85748, Germany \\ 2 California Institute of Technology, MC 105-24, 1200 East California Boulevard, Pasadena, CA 91125, USA \\ ${ }^{3}$ University of Maryland, Baltimore County, 1000 Hilltop Circle, Baltimore, MD 21250, USA \\ ${ }^{4}$ Leiden Observatory, Leiden University, P.O. Box 9513, 2300 RA, Leiden, The Netherlands \\ ${ }^{5}$ INAF Osservatorio Astronomico di Bologna, Via Ranzani 1, I-40127 Bologna, Italy \\ ${ }^{6}$ University of Zagreb, Physics Department, Bijenička cesta 32, 10000 Zagreb, Croatia

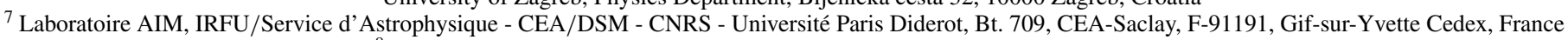 \\ ${ }^{8}$ Max-Planck-Institut für Astronomie, Königstuhl 17, D-69117 Heidelberg, Germany \\ ${ }^{9}$ Space Telescope Science Institute, 3700 San Martin Drive, Baltimore, MD 21218, USA \\ ${ }^{10}$ Excellence Cluster "Universe" and IPP-Max Planck institute for Plasma Physics Boltzmann Strasse 2, Garching 85748, Germany \\ ${ }^{11}$ Institute for Astronomy, University of Hawaii 2680 Woodlawn Drive, Honolulu, HI 96822, USA \\ ${ }^{12}$ Large Binocular Telescope Observatory, University of Arizona, Tucson, AZ 85721, USA \\ Received 2009 October 30; accepted 2010 February 19; published 2010 April 8
}

\begin{abstract}
We quantify the importance of the mechanical energy released by radio galaxies inside galaxy groups. We use scaling relations to estimate the mechanical energy released by 16 radio-active galactic nuclei located inside X-raydetected galaxy groups in the COSMOS field. By comparing this energy output to the host groups' gravitational binding energy, we find that radio galaxies produce sufficient energy to unbind a significant fraction of the intragroup medium. This unbinding effect is negligible in massive galaxy clusters with deeper potential wells. Our results correctly reproduce the breaking of self-similarity observed in the scaling relation between entropy and temperature for galaxy groups.
\end{abstract}

Key words: intergalactic medium - galaxies: active - galaxies: clusters: general - radio continuum: galaxies X-rays: galaxies: clusters

Online-only material: color figures

\section{INTRODUCTION}

Galaxy groups are important laboratories in which to investigate the importance of non-gravitational processes in structure formation. These processes are potentially more important in galaxy groups than in massive clusters because of their lower gravitational binding energy. This is suggested by the significant deviation of the observed X-ray luminosity and entropy versus temperature $\left(L_{\mathrm{X}}-T\right.$ and $\left.S-T\right)$ scaling relations in groups compared to the relation expected in a purely gravitational scenario (see also Pratt \& Arnaud 2003; Markevitch 1998; Arnaud \& Evrard 1999; Ponman et al. 2003; Sun et al. 2009; Pratt et al. 2009). Radiative cooling can be invoked to explain this deviation, but then the predicted fraction of stars in clusters of a given mass is incorrect (Voit 2005; Balogh et al. 2008). To simultaneously explain the properties of the intracluster/ group medium (ICM) and account for the observed properties of galaxies, it is necessary to take into account a major contribution to the cluster/group energetics from non-gravitational heating.

The two main sources of non-gravitational heating are star formation and active galactic nuclei (AGNs). Cosmological simulations (e.g., Kay 2004; Bower et al. 2006; Sijacki \& Springel 2006) show that both processes are required to reproduce the properties of the ICM. In particular, recent simulations by Bower et al. (2008) successfully reproduce both the galaxy and ICM properties (see Short \& Thomas 2009) when they include a "radio-mode" AGN feedback phase: in this phase the movement of bubbles inflated by the AGN jets transfers energy into the gas within the cluster (mechanical heating). The observable objects providing this type of feedback inside groups and clusters would be radio galaxies (Croton et al. 2006). The main difference between the Bower et al. (2008) model and others, including radio-mode AGNs (Bower et al. 2006; Sijacki \& Springel 2006; Puchwein et al. 2008), is that it allows the radio-mode feedback to expel gas from the X-ray-emitting regions of the system. The importance of such AGN feedback in groups could explain the observational result by Lin et al. (2003), McCarthy et al. (2007), and Giodini et al. (2009) that the total baryon fraction in groups is lower than the cosmic value estimated from cosmic microwave background (CMB) observations (see Giodini et al. 2009 for more details). The discrepancy decreases in systems of higher total mass, such that it is $<1 \sigma$ for massive clusters.

In this paper, we propose a simple, direct method to test the hypothesis that radio galaxies in groups can indeed inject enough mechanical energy to unbind the intracluster gas. The paper is structured as follows. In Section 2, we select a sample of 16 groups from the COSMOS $2 \mathrm{deg}^{2}$ survey discussed in Giodini et al. (2009), each hosting a radio galaxy within the virial radius (Schinnerer et al. 2007; Smolčić et al. 2008), plus a control sample of massive clusters from Bîrzan et al. (2004). In Sections 3 and 5, we then compare the groups' binding energy to the mechanical energy output by the radio sources, derived from their total radio luminosity through scaling relations. Applying this method, we show that the mechanical removal of gas from the group region is indeed energetically feasible for systems below $\sim 3 \times 10^{14} M_{\odot}$. In Section 6, we discuss how this scenario 
compares to the deviation in the scaling relation between entropy and temperature at the groups scale.

We adopt a $\Lambda$ CDM cosmology with $h=0.72, \Omega_{m}=0.25$, and $\Omega_{\Lambda}=0.75$.

\section{THE SAMPLES}

\subsection{Radio Galaxies in X-ray-detected Groups}

We use the catalog of 91 X-ray-selected groups from the COSMOS survey (Scoville et al. 2007a; A. Finoguenov et al. 2010, in preparation), selected as described in Giodini et al. (2009). Extended source detection was performed using a multiscale wavelet reconstruction of a mosaic of XMM-Newton and Chandra data. For each group, member galaxies are identified within $R_{500}{ }^{13}$ of the group center, utilizing the highquality photometric redshifts available $(\sigma(\Delta z) /(1+z)=0.02$ at $i_{\mathrm{AB}}{ }^{14}<25$; Ilbert et al. 2009).

We use a sub-sample of the Very Large Array (VLA)COSMOS catalog (Schinnerer et al. 2007; Smolčić et al. 2008) to identify radio galaxies lying inside the $\mathrm{X}$-ray-selected groups. Of the 60 radio galaxies ${ }^{15}$ identified within the VLA-COSMOS Large Project (Schinnerer et al. 2007; $1.49 \mathrm{GHz}$ ), about $80 \%$ have been associated with a secure optical counterpart (Smolčić et al. 2008) with $i_{\mathrm{AB}} \leqslant 26$, and accurate photometry (thus also with accurate photometric redshifts; Ilbert et al. 2009; Salvato et al. 2009).

We have cross-correlated this sample of radio galaxies with the X-ray-selected galaxy groups in three-dimensional space using a search radius of $1 \times R_{200}$ (A. Finoguenov et al. 2010, in preparation) around the groups' centers and within $0.02 \times(1+z)$ from the group's redshift. This resulted in a sample of 16 systems matched in position and redshift. In the Appendix, we show the (solid) contours of the radio $20 \mathrm{~cm}$ and X-ray emission superimposed to the SUBARU $z p$ band image for each of the groups. In nine out of 16 cases the radio galaxy is located in the core of the group (defined as $R<0.15 R_{200}$ ). The $20 \mathrm{~cm}$ radio luminosity densities ${ }^{16}$ of these galaxies range from $\sim 5.5 \times 10^{22}$ to $4.8 \times 10^{25} \mathrm{~W} \mathrm{~Hz}{ }^{-1}$, with a median luminosity of $8.9 \times 10^{24} \mathrm{~W} \mathrm{~Hz}^{-1}\left(v F_{v} \sim 7.3 \times 10^{38}-6.4 \times\right.$ $10^{41} \mathrm{erg} \mathrm{s}^{-1}$, with a median luminosity of $1.18 \times 10^{41} \mathrm{erg} \mathrm{s}^{-1}$ ). This median luminosity is at the high end of the radio luminosity distribution of the full radio AGN sample (compare to Figure 17 in Smolčić et al. 2008 and Figure 5 in Smolčić et al. 2009), consistent with previous findings that powerful radio galaxies inhabit group-scale environments (e.g., Baum et al. 1992). The redshift distribution of the 16 groups is fairly uniform between 0.1 and 1 , with the exception of six sources concentrated at $z \sim 0.3$ (where a large structure extends throughout the whole COSMOS field). The groups have $\mathrm{X}$-ray luminosities ranging from $1 \times 10^{42}$ to $8.7 \times 10^{43} \mathrm{erg} \mathrm{s}^{-1}$ and span a mass range of $2 \times 10^{13} M_{\odot}<M_{200}<2 \times 10^{14} M_{\odot}$ with a median mass of $7.14 \times 10^{13} M_{\odot}$.

\footnotetext{
$13 R_{\Delta}(\Delta=500,200)$ is the radius within which the mass density of a group/cluster is equal to $\Delta$ times the critical density $\left(\rho_{c}\right)$ of the universe. Correspondingly, $M_{\Delta}=\Delta \rho_{c}(z)(4 \pi / 3) R_{\Delta}^{3}$ is the mass inside $R_{\Delta} . M_{200}$ is computed using an $L_{X}-M_{200}$ relation established via the weak lensing analysis in Leauthaud et al. (2010). The catalog value of $M_{200}$ is converted into $M_{500}$ assuming an NFW profile with a concentration parameter computed from the mass-dependent relation of Macciò et al. (2007).

14 AB magnitude in the Subaru $i$ band.

15 The term "radio galaxy" is used here to describe an extended radio source with clear jet/lobe structure.

16 Computed using the total flux densities $\left(F_{v}\right)$. $K$-correction is also applied assuming a spectral index of $\alpha=0.7\left(F_{v} \propto v^{-\alpha}\right)$.
}

\subsection{The Comparison Sample of Massive Clusters}

The COSMOS X-ray sample is mostly composed of groups. We complement it with 12 well-known radio galaxies inside massive clusters, extracted from the sample of Bîrzan et al. (2004). We use those clusters from Birzan's sample which overlap with the HIFLUGCS survey (Reiprich \& Boehringer 2002) so that we can use the X-ray parameters determined from the HIFLUGCS clusters. In addition, we require that the radio source within those clusters is associated with a secure near-IR (NIR) counterpart in the Two Micron All Sky Survey (2MASS) catalog (Skrutskie et al. 2006). These requirements eliminate four of the clusters in the original Birzan et al. sample. Each of these clusters contains X-ray cavities associated with radio bubbles likely connected with AGNs activity of the central galaxy. The radio galaxies have been identified within the NRAO VLA Sky Survey (NVSS) at $1.49 \mathrm{GHz}$ (Condon et al. 1998), except in the case of the Centaurus Cluster where data come from the 1.41 GHz Parkes Radio Sources Catalog (Wright \& Otrupcek 1990). The $20 \mathrm{~cm}$ radio luminosities of the radio galaxies range between $2 \times 10^{39}$ and $2 \times 10^{43} \mathrm{erg} \mathrm{s}^{-1}$, with a median luminosity of $1.4 \times 10^{42} \mathrm{erg} \mathrm{s}^{-1}$, more than 10 times higher than the median radio luminosity of the radio galaxies in the COSMOS sample.

The X-ray parameters for these clusters are provided by the X-ray analysis in the HIFLUGCS survey and converted for the standard cosmology used in this paper. The sample consists of very local clusters, ranging in total mass between $1 \times 10^{14} M_{\odot}<M_{200}<1.2 \times 10^{15} M_{\odot}$ with a median mass of $4.25 \times 10^{14} M_{\odot}$, almost 10 times higher than the median total mass of the systems in the COSMOS sample.

\section{ANALYSIS OF COSMOS GROUP SAMPLE}

\subsection{Mechanical Energy Input by Radio Galaxies in Groups}

We estimate the mechanical energy input by a radio galaxy into the ICM over the group lifetime from the mechanical luminosity of the radio source multiplied by the fraction of time a massive galaxy spends in the radio-AGN phase. The mechanical luminosity for the radio galaxies in our sample is estimated from the scaling relation presented in Bîrzan et al. (2008). These authors studied a sample of galaxy clusters showing signatures of cavities and bubbles in the X-ray surface brightness twodimensional distribution, with a powerful radio source as a central galaxy. The cavity power of the radio source, estimated from the $p d V$ work of the jet/lobe on the surrounding ICM, is found to be correlated (albeit with a large scatter) with the monochromatic radio power at $1.49 \mathrm{GHz}$ of the central galaxies $\left(P_{1.49 \mathrm{GHz}}\right)$ as

$$
P_{\text {cav }} \propto P_{1.49 \mathrm{GHz}}^{0.35 \pm 0.07}
$$

(see Equation (16) in Bîrzan et al. 2008). $P_{1.49 \mathrm{GHz}}$ is computed from the radio emission of the entire source. This estimate is a lower limit to the mechanical luminosity of the AGN outbursts, since it does not take into account the energy dissipated (e.g., in shocks). $P_{\text {cav }}$ is related to the $p d V$ work through

$$
P_{\mathrm{cav}}=\frac{4 P V}{\tau}
$$

(Churazov et al. 2002; Bîrzan et al. 2008), where $\tau$ is the duration of each single AGN outburst and 4 is the factor used for relativistic plasma. Smolčić et al. (2009) investigated the fraction of radio AGNs as a function of cosmic time and stellar mass of the galaxy. This fraction can be related, through a 
Table 1

Mechanical and Binding Energy for 16 COSMOS X-ray Groups

\begin{tabular}{|c|c|c|c|c|c|c|c|c|c|}
\hline XID & $\begin{array}{c}\text { R.A. } \\
{[\mathrm{J} 2000]}\end{array}$ & $\begin{array}{c}\text { Decl. } \\
{[\mathrm{J} 2000]}\end{array}$ & $z$ & $\begin{array}{c}P_{1.49 \mathrm{GHz}} \\
\left(10^{24} \mathrm{~W} / \mathrm{Hz}\right)\end{array}$ & $\begin{array}{c}P_{\text {cav }} \\
\left(10^{36} \mathrm{~W}\right)\end{array}$ & $\begin{array}{c}E_{\text {binding }} \\
\left(10^{60} \mathrm{erg}\right)\end{array}$ & $\begin{array}{c}E_{\text {mech }} \\
\left(10^{60} \mathrm{erg}\right)\end{array}$ & $\begin{array}{l}\tau_{\text {radio }} \\
(\mathrm{Gyr})\end{array}$ & $R / R_{200}$ \\
\hline 107 & 149.60965 & 2.14799 & 0.28 & 1.11 & 7.353 & $5.916_{1.937}^{2.342}$ & $0.514_{0.441}^{3.087}$ & 0.221 & 0.3783 \\
\hline 262 & 149.60007 & 2.82118 & 0.34 & 19.0 & 19.85 & $127.9_{26.49}^{38.80}$ & $24.15_{20.70}^{144.9}$ & 3.858 & 0.0011 \\
\hline 253 & 149.75626 & 2.79472 & 0.49 & 6.71 & 13.78 & $40.16_{9.721}^{12.26}$ & $10.14_{8.693}^{60.85}$ & 2.332 & 0.0042 \\
\hline 246 & 149.76132 & 2.92909 & 0.34 & 0.90 & 6.828 & $289.8_{67.46}^{76.73}$ & $7.003_{6.003}^{42.02}$ & 3.252 & 0.6181 \\
\hline 311 & 149.93796 & 2.60627 & 0.34 & 6.38 & 13.54 & $17.27_{3.907}^{4.792}$ & $0.183_{0.157}^{1.101}$ & 0.042 & 0.2195 \\
\hline 264 & 149.99847 & 2.76914 & 0.16 & 0.32 & 4.775 & $6.940_{2.247}^{3.179}$ & $0.004_{0.003}^{0.027}$ & 0.003 & 0.0007 \\
\hline 281 & 150.08617 & 2.53141 & 0.88 & 8.90 & 15.21 & $84.94_{22.45}^{27.05}$ & $\begin{array}{l}1.241_{1.064}^{7.450} \\
\text { - }\end{array}$ & 0.258 & 0.8614 \\
\hline 191 & 150.11434 & 2.35651 & 0.22 & 1.71 & 8.554 & $8.380_{2.150}^{2.959}$ & $6.122_{5.248}^{36.73}$ & 2.269 & 0.0757 \\
\hline 237 & 150.11774 & 2.68425 & 0.34 & 27.7 & 22.65 & $105.1_{23.49}^{31.65}$ & $19.56_{16.77}^{117.4}$ & 2.738 & 0.0027 \\
\hline 29 & 150.17996 & 1.76887 & 0.34 & 30.0 & 23.29 & $58.49_{12.77}^{16.80}$ & $31.63_{27.11}^{189.8}$ & 4.306 & 0.0016 \\
\hline 64 & 150.19829 & 1.98628 & 0.43 & 12.4 & 17.11 & $21.10_{4.941}^{7.358}$ & $18.06_{15.48}^{108.4}$ & 3.348 & 0.0030 \\
\hline 35 & 150.20661 & 1.82327 & 0.52 & 10.2 & 16.00 & $30.87_{8.342}^{11.07}$ & $15.32_{13.13}^{91.95}$ & 3.037 & 0.0008 \\
\hline 6 & 150.28821 & 1.55571 & 0.36 & 1.13 & 7.401 & $77.91_{17.35}^{20.23}$ & $0.070_{0.060}^{0.425}$ & 0.030 & 0.2279 \\
\hline 149 & 150.41566 & 2.43020 & 0.12 & 0.05 & 2.564 & $50.31_{10.32}^{14.98}$ & $0.002_{0.002}^{0.014}$ & 0.003 & 0.1957 \\
\hline 40 & 150.41386 & 1.84759 & 0.96 & 48.5 & 27.54 & $108.1_{24.40}^{34.85}$ & $1.786_{1.530}^{10.71}$ & 0.205 & 0.4888 \\
\hline 120 & 150.50502 & 2.22506 & 0.83 & 16.4 & 18.88 & $425.0_{90.06}^{112.5}$ & $24.92_{21.36}^{149.5}$ & 4.185 & 0.0267 \\
\hline
\end{tabular}

Note. For each of the 16 COSMOS groups, the columns indicate (1) X-ray catalog ID number, (2) R.A., (3) decl., (4) redshift, (5) power at 1.4 GHz, (6) mechanical power, (7) binding energy $\pm 1 \sigma$ confidence limit, (8) $E_{\text {mech }} \pm 1 \sigma$ confidence limit, (9) $\tau_{\text {radio }}$, and (10) distance from the center.

probability argument detailed in Smolčić et al. (2009), to the time a galaxy of a given stellar mass and at a given redshift spends as a radio galaxy $\left(\tau_{\text {radio }}\right)$. Using this result, we can estimate the average duration of radio sources as a function of redshift and stellar mass of the host galaxy (see Figure 12 in Smolčić et al. 2009). ${ }^{17}$ This gives a plausible timescale during which the radio AGN can have injected mechanical energy into its environment. For the 16 COSMOS X-ray-selected groups, $\tau_{\text {radio }}$ ranges between 0.003 and $4.18 \mathrm{Gyr}$, with a median value of 3.1 Gyr. The mechanical energy contribution can then be estimated as

$$
E_{\text {mech }}=P_{\text {cav }} \times \tau_{\text {radio }} .
$$

The values of $E_{\text {mech }}$ for our sources are shown in Table 1 and span a range between $\sim 2 \times 10^{57}-3 \times 10^{61}$ erg $h_{72}^{-2}$. The uncertainties in the radio mechanical energy input are dominated by the scatter in the scaling relation used to convert the monochromatic power into mechanical luminosity, which amounts to $0.85 \mathrm{dex}$, and by the uncertainties on $\tau_{\text {radio }}$. We use $\tau_{\text {radio }}$ as derived from an average estimate over a sample of radio galaxies in the COSMOS field as a whole, irrespective of their environment. One might expect the density of the environment surrounding the jets to have a significant impact on the jet lifetime. However, the fraction of radio galaxies that resides within the COSMOS groups is comparable with the fraction of red massive galaxies within groups in the control sample used in Smolčić et al. (2009; respectively $18 \%$ and $16 \%$ within $R_{200}$ ); this assures that the statistical argument used to compute the timescales also holds in this case. Furthermore, we can estimate

\footnotetext{
17 To derive the values of $\tau_{\text {radio }}$ Smolčić et al. (2009), it is assumed that the radio parent population (red massive galaxies) is formed at $z=3$ (Renzini 2006) and survives until $z=0$. Since the COSMOS radio galaxies are not at $z=0$, the timescales computed in Smolčić et al. (2009) coincide with ours if multiplied by $\frac{t(z=z g a l)-t(z=3.0)}{10^{9} \mathrm{yr}}$, where $t$ is the age of the universe at redshift $z$ and zgal is the redshift of the radio galaxy.
}

an average timescale based on only extended radio galaxies in the whole COSMOS group sample as follows. Of the 141 COSMOS groups at $z<1$ and with $L_{\mathrm{X}}>10^{42} \mathrm{erg} \mathrm{s}^{-1}, 32$ contain a multi-component radio galaxy. Therefore, the average duration of the radio galaxy activity during this time interval is $(32 / 141) \times(t(z=1)-t(z=0))$. This is $\sim 1.7 \mathrm{Gyr}$, a timescale comparable with the average lifetime estimated with the method by Smolčić et al. (2009; 1.6 Gyr). ${ }^{18}$

\subsection{Binding Energy of the Intragroup Medium}

We consider the shape of the dark matter halos to be characterized by Navarro-Frenk-White (NFW; Navarro et al. 1996) radial profiles

$$
\rho(x)=\frac{\rho_{\text {crit }} \delta_{c}}{x(x+1)^{2}},
$$

where $x=r / r_{s}, r_{s}$ is the characteristic radius, and $\rho_{c}$ is the critical density of closure of the universe. $\delta_{c}$ is defined as

$$
\delta_{c}=\frac{200 c^{3}}{3 \ln (1+c)-c /(1+c)},
$$

and $c$ is the concentration of the halo. The scale radius and the concentration are linked by the relation $r_{s}=R_{500} / c_{500}$, where $c_{500}$ is the dark matter concentration inside $R_{500}$. We estimate the binding energy out to $R_{500}$ because the kinetic energy of the infall velocity field along filaments becomes important beyond this radius (Evrard et al. 1996) and our simple model may not then be applicable. Furthermore, we can evaluate reliable gas masses from the X-ray observations only within $R_{500}$. For simplicity, we assume that the gas follows the same distribution as the dark matter. We define as binding energy the

\footnotetext{
18 Computed as $\frac{\max \left(\tau_{\text {radio }}\right)-\min \left(\tau_{\text {radio }}\right)}{2}$.
} 
total potential energy needed to push the ICM gas inside $R_{500}$ beyond $R_{200}$. The binding energy is computed as

$$
\begin{aligned}
E_{\text {binding }} & =\int_{0}^{M_{g, 500}}\left[\phi(r)-\phi\left(R_{200}\right)\right] d M_{g} \\
& =4 \pi \int_{0}^{R_{500}} \phi(r) \rho_{g}(r) r^{2} d r .
\end{aligned}
$$

We neglect the additive constant given by the term $\phi\left(R_{200}\right)$, as it is small with respect to the other terms of the equation. We use the definition of gas mass within $R_{500}$ as

$$
M_{g}=M_{g}\left(R_{500}\right)=4 \pi \int_{0}^{R_{500}} \rho_{g}(r) r^{2} d r .
$$

The potential of a spherical NFW model is (Hayashi et al. 2007)

$$
\phi(r)=A \times \frac{\ln (1+x)}{x},
$$

where $A$ is

$$
A=-\frac{G M_{200}}{r_{s}(\ln (1+c)-c /(1+c))} .
$$

Thus, substituting the terms into Equation (6), we compute the binding energy of the ICM gas in an NFW dark matter halo as follows:

$$
E_{\text {binding }}=f_{\text {gas }} 4 \pi \rho_{\text {crit }} \delta_{c} A r_{s}^{3} \int_{0}^{c_{500}} \frac{\ln (1+x)}{(1+x)^{2}} d x,
$$

where $f_{\text {gas }}$ is the gas fraction. The concentration parameter for the COSMOS groups has been computed from the massdependent relation of Macciò et al. (2007). The error bars on the binding energy are estimated using a Monte Carlo method to numerically propagate the errors on $M_{200}$ and $R_{200}$, the scatter in the $c-M_{200}$ and in the $f_{\text {gas }}-M_{500}$ relation.

We cannot estimate the gas masses from most of the existing $\mathrm{X}$-ray observations of the COSMOS X-ray-selected groups because of insufficient signal-to-noise ratio. We therefore estimate the gas fraction in the groups from the mean trend of the gas mass fraction as a function of $M_{500}$. This trend was established from an independent compilation of high-quality observations of local $(z<0.2)$ groups and clusters in the same mass range as the sample under consideration here (Pratt et al. 2009). The observed relation $\left(f_{\text {gas }} \propto M_{500}^{0.21}\right)$ suggests that lower mass systems have proportionally less gas than high-mass systems.

\section{ANALYSIS OF GALAXY CLUSTER SAMPLE}

\subsection{Mechanical Energy Input by Radio Galaxies in Massive Clusters}

In order to compare the energy input from radio galaxies in groups and clusters, we include in our analysis a sample of well-known radio galaxies in massive clusters, extracted from the sample of (Bîrzan et al. 2004; see Section 2.2). We use their tabulated value of $p d V$ to compute the mechanical energy input over the average time the galaxy has spent as a radio galaxy. Birzan et al. provide a value for the energy input for both filled and radio ghost cavities. In order to obtain a measure of the average input, we sum the $p d V$ for all the cavities in a cluster and multiply it by the number of events (i.e., how often the radio jet was turned on). The latter is given by the ratio between $\tau_{\text {radio }}$ and the duration of a single radio event (assuming that all the active AGN phases have the same duration).

We choose the oldest cavity's age as an indication of the duration of the radio event. Bîrzan et al. (2004) calculate the age of each cavity in three ways: (1) the time required for the cavity to rise at the sound velocity, (2) the time required for the bubble to rise buoyantly at the terminal velocity, and (3) the time required to refill the displaced volume. We adopt the average of the three age estimates; this is generally similar to the age computed for a buoyantly rising bubble. We take the error on the cavity's age to be the difference between the shortest and longest lifetime estimated via the three different methods.

As $\tau_{\text {radio }}$ for our sample is derived following Smolčić et al. (2009), it depends on the redshift and the stellar mass of the radio galaxy. We computed the stellar masses for the central radio galaxy in the massive cluster sample using the $K$-band photometry provided by the 2MASS survey (Skrutskie et al. 2006). This method is robust, since radio galaxies contain mostly type 2 (obscured) AGNs, whose emission does not significantly contaminate the optical-NIR part of the galaxy spectrum. We assume an $M / L_{K}$ ratio for a stellar population with an age of $\sim 10 \mathrm{Gyr}$ (corresponding to the age of the stars in a galaxy at $z \sim 0$ ), obtained by Drory et al. (2004; $M / L_{K}=1.4$ with a Salpeter IMF). The quoted error on the $M / L_{K}$ in Drory et al. (2004) is $25 \%-30 \%$; a change in stellar mass of this magnitude does not affect significantly the timescales, we estimate. The stellar masses are then converted to a Chabrier IMF by subtracting an offset of 0.2 dex.

\subsection{Binding Energy of the Intracluster Medium}

We compute the binding energy for the Birzan et al. clusters in the same way as for the COSMOS groups, using the value of $M_{200}$ and $R_{200}$ provided by the X-ray analysis in the HIFLUGCS survey (Reiprich \& Boehringer 2002). We assume a constant concentration parameter of 5. Errors on $E_{\text {binding }}$ are propagated numerically via a Monte Carlo method, in the same way as the COSMOS groups (see Section 3.2). When computing the binding energy of clusters individually, we also test the cluster result using the scaling relations adopted for the COSMOS groups, both for computing $M_{200}$ (Leauthaud et al. 2010) and for estimating their mechanical energy output (Bîrzan et al. 2008). The change in our calculations does not qualitatively affect our results. The values of $E_{\text {binding }}$ change by less than a factor of 2 on average, while values of $E_{\text {mech }}$ are perturbed randomly within the error bars.

\section{RESULTS}

\subsection{The Balance of Radio Input and Binding Energy}

Figure 1 shows the binding energy of the gas versus the energy output from radio galaxies. In the group regime, the two energies span a comparable range of values $\left(10^{58}-10^{61} \mathrm{ergs}\right)$, while for clusters the binding energy exceeds the total mechanical output of radio galaxies by a factor of $\sim 10^{2}-10^{3}$. In particular, for seven groups, the two energies are consistent at the $1 \sigma$ level, and for all other groups except two the equality holds at $3 \sigma$, meaning that radio galaxies potentially provide sufficient energy to unbind the gas in a large fraction of these groups. It is interesting to note that, in all the groups with $E_{\text {mech }} \sim$ $E_{\text {binding, }}$, the radio galaxy lies within $0.15 \times R_{200}$ from the center of the group. This suggests that a radio galaxy in a group is most likely to input sufficient energy into the ICM 


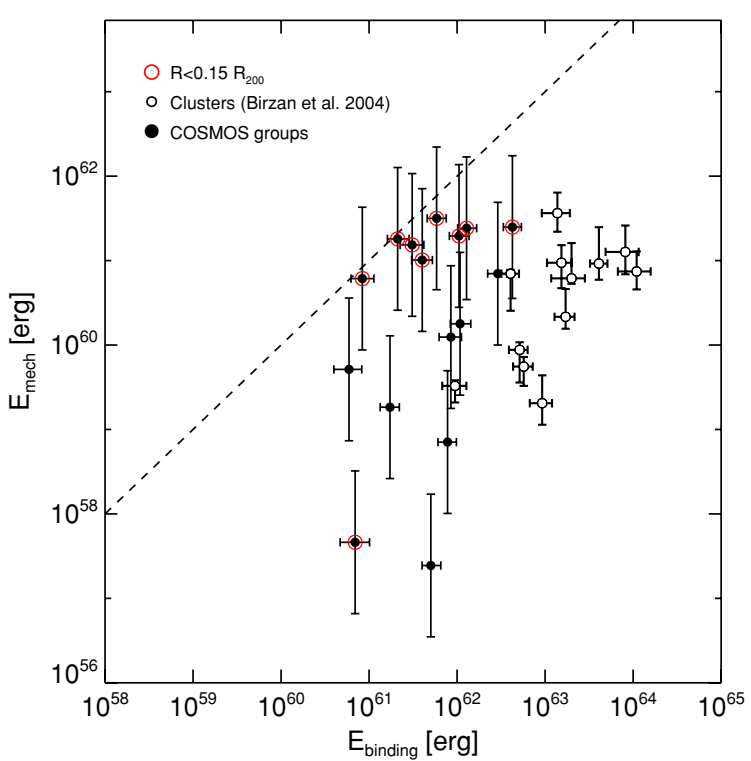

Figure 1. Binding energy of the cluster/group gas vs. the output mechanical energy from radio galaxies. Solid points show the $16 \mathrm{X}$-ray-selected groups in the COSMOS field that host a radio galaxy within their virial radius. Large concentric circles indicate groups that host a radio galaxy within the core ( $\left.R \leqslant 0.15 \times R_{200}\right)$. Open points show the sample of massive local clusters drawn from Bîrzan et al. (2004). The dashed line shows equality. The binding energy in clusters exceeds the total mechanical output by a factor of $\sim 10^{2}-10^{3}$. In all cases, except one where a radio galaxy lies in the center of a group, the mechanical energy output from the radio galaxy is of the same order as the binding energy for the COSMOS groups analyzed here.

(A color version of this figure is available in the online journal.)

to unbind a part of the gas if it lies at the core of the group. Moreover, radio sources outside the group core reside in lower density environments, and our calculations of those binding energies may be overestimates. The different energy balance in groups and clusters demonstrates the importance of AGN heating in groups and shows that the mechanical removal of gas from groups is energetically possible. This has important consequences for the understanding of the baryonic budget in these systems (see Giodini et al. 2009).

\subsection{Can Radio Galaxies Offset Radiative Cooling in Galaxy Groups?}

We now compare the mechanical energy input by radio galaxies with the energy required to offset the cooling in the group center $\left(E_{\text {cool }}\right)$. As detailed in Fabian et al. (1994), Peterson et al. (2003), and McNamara \& Nulsen (2007), the cooling time in cluster/group centers can be lower than the Hubble time, implying that large reservoirs of cold gas could accumulate in these regions. However, evidence that the gas does not cool below approximately one-third of the virial temperature (Kaastra et al. 2004) indicates the presence of a heat source providing enough energy to offset the cooling. Several studies (e.g., Peterson et al. 2003; Peterson \& Fabian 2006; McNamara \& Nulsen 2007) suggest AGN feedback as a viable heating source. To test this hypothesis, we check whether the cooling energy is lower than the mechanical energy of the rising bubbles. We estimate $E_{\text {cool }}$, assuming that the time during which the gas has been cooling is equal to the lifetime of the group, which we assume to be 5 Gyr (Voigt \& Fabian 2004). The cooling energy can then be estimated as

$$
E_{\mathrm{cool}}=L_{\mathrm{cool}} \times t_{v}=f_{\mathrm{cool}} L_{\mathrm{bol}} \times t_{v}
$$

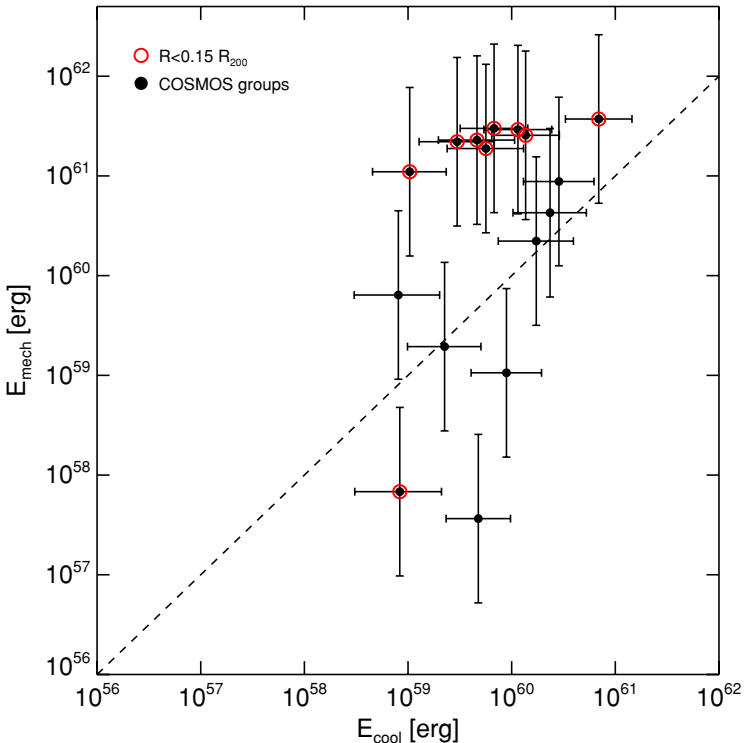

Figure 2. Output mechanical energy from radio galaxies $\left(E_{\text {mech }}\right)$ vs. energy radiated inside the cooling radius $\left(E_{\mathrm{cool}}\right.$; i.e., energy required to offset the cooling in the group center) for $16 \mathrm{X}$-ray-selected groups from the COSMOS survey. The dashed line shows the equality line. Large concentric circles mark the radio-galaxies inside $0.15 \times R_{200}$. Uncertainties on $E_{\text {cool }}$ are computed allowing an error of a factor of 2 on $f_{\text {cool }}$.

(A color version of this figure is available in the online journal.)

where $t_{v}$ is the lifetime of the group, and $f_{\text {cool }}$ is the fraction of bolometric luminosity assumed to be emitted inside the cooling radius (where the cooling time of the gas is lower than the Hubble time). In general, this contribution is found to be $\gtrsim 10 \%$ of the total cluster X-ray luminosity (McNamara \& Nulsen 2007). Also, the scatter in the $L_{X}-T$ scaling relation due to the contribution of cool core clusters can be up to a factor of 2 (Chen et al. 2007; Pratt et al. 2009). Given these considerations, we assume that $25 \%$ of the total bolometric X-ray luminosity is emitted inside the cooling radius (Peres et al. 1998). Since the relative contribution of the cool core to the total X-ray luminosity is higher in groups than in massive clusters, this value is a good estimate of the average contribution of the cooling core to the total luminosity of a group.

In Figure 2, we compare $E_{\text {mech }}$ and $E_{\text {cool }}$ in our groups. The mechanical energy injected by all but one of the core radio galaxies is higher than the radiative losses, and exceeds $E_{\text {cool }}$ by an order of magnitude in several cases. We can thus conclude that radiative losses do not greatly affect the net energy output of radio galaxies in the cores of groups. On the other hand, the mechanical output by non-central radio galaxies is typically of the same order as $E_{\text {cool }}$. Moreover, these sources reside mostly outside the cooling radius $\left(\sim 0.15 R_{200}\right)$, where the cooling time is higher than the Hubble time. In this location, the gas does not lose as much energy through radiative cooling as in the core of the group, so these galaxies do not provide the required feedback at the right location.

\subsection{Impact of Systematic Effects}

The above calculations rest on several assumptions and should be regarded as rough estimates. One critical simplification is the calculation of the lifetime of a radio galaxy: the statistical argument used in Smolčić et al. (2009) relies on knowledge about the parent population that hosts the radio galaxies. In the absence of evidence in contrast, we assume that there is 
no significant difference between the radio galaxy elliptical hosts in groups and in low-density environments (Feretti \& Giovannini 2008). We note that even if $\tau_{\text {radio }}$ were incorrect by a factor of 4 , the mechanical output in clusters would still be significantly lower than the binding energy, but would remain consistent with the binding energy for many of the groups (see Figure 1).

Other biases may arise from the scaling relation of Birzan et al., which we use to compute the mechanical energy: the large scatter in the $P_{1.49 \mathrm{GHz}}-P_{\text {cav }}$ relationship (0.85 dex) means that care must be taken when using the inferred value as the mean mechanical energy, since most of our calculations rest on the assumption that over the cluster/group lifetime each burst has on average the same power. Indeed, Nipoti \& Binney (2005) suggested that the distribution of the outbursts over the cluster/ group lifetime is log normal rather than Gaussian; therefore, in any system there would be a good chance of observing smaller than average jet powers. Instead, much of the power would be generated by a rare, more powerful outburst, such as that observed in MS $0735+7421$ by Gitti et al. (2007). These arguments rest on the assumption that the observed scatter in $P_{1.49 \mathrm{GHz}}-P_{\text {cav }}$ in the observed ensemble of clusters is a good description of the time variability of the AGN power in individual objects. In general, the ensemble scatter is an upper limit to the scatter in the time variability. If we assume this scatter to represent also for the time variability, we are statistically underestimating the mechanical energy output over the group lifetime by a factor that we compute as follows. The scatter in the Birzan et al. relationship ( $0.85 \mathrm{dex}$ ) corresponds to a probability $\gtrsim 80 \%$ of observing a value smaller than the mean from a single observation (compare to Nipoti \& Binney 2005). Thus, if we assume that the observed value of $P_{1.49 \mathrm{GHz}}$ scatters around the median of the distribution, the ratio between the median and the mean for a log-normal distribution (which depends only on the scatter $\sigma$ ) tells us the scaling factor for the "true" mean mechanical energy:

$$
\frac{\text { mean }}{\text { median }}=\frac{e^{\sigma^{2}}}{2}=6.8 .
$$

Therefore, the typical observed mechanical power may be underestimated by a factor $\leqslant 7$ with respect to the mean. This value, though not negligible, goes in the direction of further increasing the mechanical output, confirming the effect we found.

Furthermore, if the bubble were over pressured when compared to the surrounding ICM (Heinz et al. 1998), the expanding bubble would carry a shock and the mechanical power may be underestimated, as well as reported by Bîrzan et al. (2004). This effect would also boost the mechanical energy to higher values, further strengthening our results.

We have also used preliminary results from VLA $324 \mathrm{MHz}$ data (V. Smolčić et al. 2010, in preparation) to double-check our estimates of the mechanical energy output from radio galaxies. Only 12 of the 16 radio galaxies are detected in the $324 \mathrm{MHz}$ band and, in all these cases, $E_{\text {mech }}$ computed using these data (using Equation (15) in Bîrzan et al. 2008) is consistent within the error bars with the value computed at $1.49 \mathrm{GHz}$. As a further check, the total radio luminosity can be computed with higher precision from break frequencies for seven of the 16 sources, using the Myers \& Spangler (1985) approximation. The value of $E_{\text {mech }}$ obtained with this improved method is consistent within the error bars with that obtained using monochromatic data.

\section{DISCUSSION: THE ENTROPY IN X-ray GROUPS}

The injection of energy by radio galaxy activity into the ICM modifies the thermodynamical state of the gas, raising the entropy $(S)$ by a significant amount compared to that generated by gravitational collapse. We define the entropy as

$$
S \propto \frac{k T}{n_{e}^{\frac{2}{3}}},
$$

where $T$ is the gas temperature in $\mathrm{keV}$, and $n_{e}$ is the gas electron density (Voit 2005). An excess entropy of $50-100 \mathrm{keV} \mathrm{cm}^{-2}$ is indeed observed at the group regime, causing a deviation from the $S-T$ relation (Ponman et al. 2003). The excess entropy is measured in the central regions (at $0.1 R_{200}$ ). In the following, we make an order of magnitude calculation of the excess entropy generated by the energy injected, then compare it with that observed in groups and predicted from the theory. We recompute the expected $S-T$ relation taking into account this excess energy, and compare it with the observational constraints of Ponman et al. (2003).

The change in entropy caused by injection of energy under constant pressure is

$$
\Delta S=\frac{2}{5} \frac{\Delta E}{n_{e}} \frac{\gamma_{T}^{5 / 3}-1}{\gamma_{T}-1}
$$

(Lloyd-Davies et al. 2000), where $\Delta E$ is the injected energy per particle, $\gamma_{T}$ is the ratio between the initial and final temperatures (a value between 1.1 and 2.0; Lloyd-Davies et al. 2000), and $n_{e}$ is the initial electron density $\left(n_{e}=10^{-2}\right.$ assuming the energy is deposited in the cluster core; e.g., Sanderson \& Ponman 2003). We compute $\Delta E$ from the mechanical energy input of radio galaxies as follows:

$$
\Delta E=E_{\mathrm{mech}} \times \frac{m_{p} \mu}{M_{\mathrm{gas}}},
$$

with $M_{\text {gas }}=f_{\text {gas }} M_{200}$, where $f_{\text {gas }}$ is estimated from the relation between gas fraction and total mass in Pratt et al. (2009). This calculation does not depend on the details of the energy injection process. We obtain values of excess entropy between 10 and $60 \mathrm{keV} \mathrm{cm}^{-2}$. This is a rough calculation but predicts values similar to those in Voit \& Donahue (2005). These authors show that an additional energy input episodic on $10^{8} \mathrm{yr}$ timescale is needed to explain the excess entropy found observationally in the core of clusters (Ponman et al. 2003; Donahue et al. 2005). The additional energy produces an entropy pedestal: Voit (2005) calculates $10 \mathrm{keV} \mathrm{cm}^{-2}$ to be the minimum entropy boost needed to explain observations, and he predicts it to be larger for groups.

The mechanical energy injected by radio galaxies into the 16 COSMOS X-ray-selected groups is roughly independent on the group mass (see Figure 1). This is not unexpected, since the black hole masses (which are a zeroth-order indicator of the mechanical energy output; Merloni \& Heinz 2007) range only between $10^{8}$ and $10^{9} M_{\odot}$ in radio galaxies (see Figure 7 in Smolčić et al. 2009). At the cluster regime the assumption that the mechanical energy is independent from cluster mass may not be entirely adequate. Indeed, Chen et al. (2007) infer, from the strength of clusters' cooling cores, that a mechanical input higher than anything observed in groups is necessary to balance the cooling of the gas in the strong cool core clusters. However, it has been shown by the same authors that much ( 90\%) of that energy input would be radiated away to balance the cooling, 


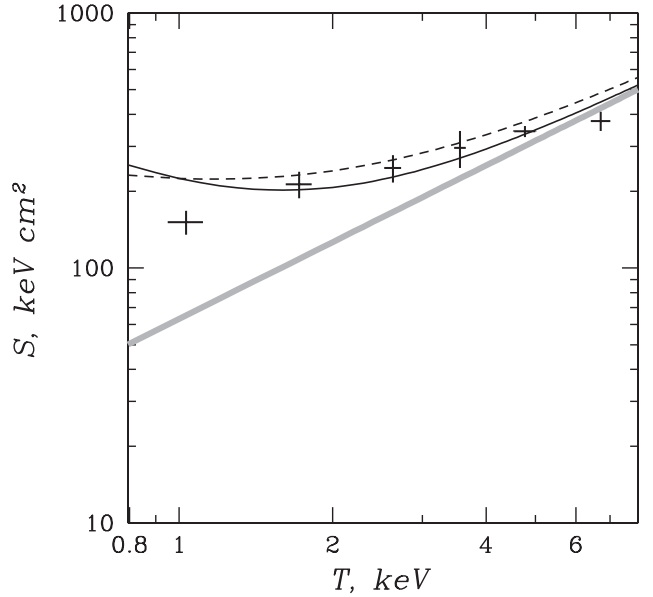

Figure 3. Scaling relation between entropy $(S)$, measured at $0.1 R_{200}$, and temperature $(T)$. The solid black line is the inferred relation accounting for a constant energy excess injected by radio galaxies (see the text for details). The gray line is the expected self-similar relation. The points show the binned means from the observations by Ponman et al. (2003). The dashed line is the same as the solid line but considering the self-similar scaling $M_{\mathrm{gas}} \propto T^{1.5}$.

and therefore would not participate to the mechanical removal of the gas.

From these considerations, we can predict how the scaling relation between entropy and temperature is affected by the injection of a constant excess energy by radio galaxies. As shown in Finoguenov et al. (2008), the energy deposition into the ICM $(\Delta E)$ is proportional to the change in entropy $\frac{\Delta S}{S}$ for a given typical $n_{e}$. We use $n_{e}=10^{-2}$ as the typical value of the density within $0.1 R_{200}$, where the majority of the energy is deposited (deposition radius; Sanderson \& Ponman 2003). Using the scaling of $M_{\mathrm{gas}} \propto T^{2}$ and $E_{\mathrm{mech}}=$ const, then

$$
\frac{\Delta S}{S} \propto \frac{E_{\mathrm{mech}}}{M_{\mathrm{gas}}} \propto \frac{C}{T^{2}},
$$

where $C$ is a constant and $M_{\text {gas }}$ is the mass of the gas within the deposition radius. We can then infer the functional dependence of $S$ on the virial temperature of the ICM as

$$
S=S_{0}+\Delta S=S_{0} \times\left(1+\frac{\Delta S}{S_{0}}\right) \propto\left(T_{0}+\frac{C}{T_{0}}\right),
$$

where $S_{0}$ and $T_{0}$ are, respectively, the entropy and the temperature of the gas before the injection of energy from a radio galaxy. The value of $C$ is computed using Equation (3) in Finoguenov et al. (2008) and has a median value of 2.56 if the energy is deposited inside the cooling radius. We assume the cooling radius to be $0.10 R_{200}$ (e.g., Ponman et al. 2003) and show the inferred functional form of $S(T)$ in Figure 3. Remarkably, the shape of the resulting scaling relation (solid line) deviates from the self-similar one (dashed line) around $\sim 4 \mathrm{keV}$, in agreement with the observed scaling relation measured at $0.1 R_{200}$ by Ponman et al. (2003, black crosses; these points are binned means). The deviation of the $\sim 1 \mathrm{keV}$ point indicates that a lower excess entropy is needed to explain very cold groups. This can be achieved requiring that the mechanical energy is deposited at a larger radius in these groups. Indeed, if the deposition radius increases, $M_{\text {gas }}$ within this radius also increases. Therefore, using Equation (16) we would obtain lower values of $\frac{\Delta S}{S_{0}}$ (and thus entropy) for these groups, matching eventually the observational point of Ponman et al. (2003) at $\sim 1 \mathrm{keV}$; if this is the case, it would confirm that the effect of feedback is more global in groups than in clusters (compare to De Young 2010). Therefore, the injection of an excess energy that is independent of groups' mass, and thus temperature (as we observe from radio galaxies in the COSMOS groups), correctly predicts the deviation of the observed $S-T$ relation from the purely gravitational relation at the group scale.

\section{CONCLUSIONS}

In this paper, we have quantified the importance of the mechanical energy input by radio galaxies inside galaxy groups. In particular, we report a striking difference between clusters and groups of galaxies; while the binding energy of the ICM in clusters exceeds the mechanical output by radio AGNs, the two quantities are of the same order of magnitude in groups that host a radio galaxy within $0.15 R_{200}$. This suggests that, while clusters can be mostly considered to be closed systems, the mechanical removal of gas is energetically possible from groups. This has implications that help explain recent findings on the baryonic fraction in groups of galaxies. Giodini et al. (2009) reported a $\sim 30 \%$ lack of gas in groups compared with the cosmological baryon mass fraction evaluated from the 5 year Wilkinson Microwave Anisotropy Probe (Dunkley et al. 2009). It has been suggested that this gas has been removed by AGN feedback.

This is consistent with cosmological models in which feedback from radio galaxies is invoked to successfully explain galaxy group/cluster properties. Based on a well-selected sample of galaxy groups and clusters that host radio galaxies, we have observationally shown for the first time that this scenario is energetically feasible. We have further shown that a constant injection of excess energy by radio galaxy naturally reproduces the self-similar breaking observed in the scaling relation between the entropy and temperature of groups.

S.G. acknowledges support by the DFG Cluster of Excellence "Origin and Structure of the universe" (http://www.universecluster.de). S.G. thanks A. Merloni for helpful comments. V.S. acknowledges support from the Owens Valley Radio Observatory, which is supported by the National Science Foundation through grant AST-0838260. V.S. and A.O. thank Unity through Knowledge Fund (http://www.ukf.hr) for collaboration support through the "Homeland Visit" grant. A.O. thanks California Institute of Technology for generous support through NASA grants 1292462 and 1344606 . We acknowledge the contributions of the entire COSMOS collaboration; more informations on the COSMOS survey are available at http://www.astro.caltech.edu/ cosmos. The authors acknowledge the National Radio Astronomy Observatory for support. The National Radio Astronomy Observatory is a facility of the National Science Foundation operated under cooperative agreement by Associated Universities, Inc.

\section{APPENDIX}

\section{RADIO IMAGES}

Figure 4 presents the contours maps of the radio $20 \mathrm{~cm}$ emission (solid lines) superimposed to the SUBARU $z p$ band image for each of the groups listed in Table 1 . Images are $3 \times 3$ arcmin and centered on the group center, except XID246, which is $4 \times 4$ arcmin wide and offset from the center group because of its location on the edge of the Subaru field coverage. The white dashed line shows the contours of X-ray flux significance. The 

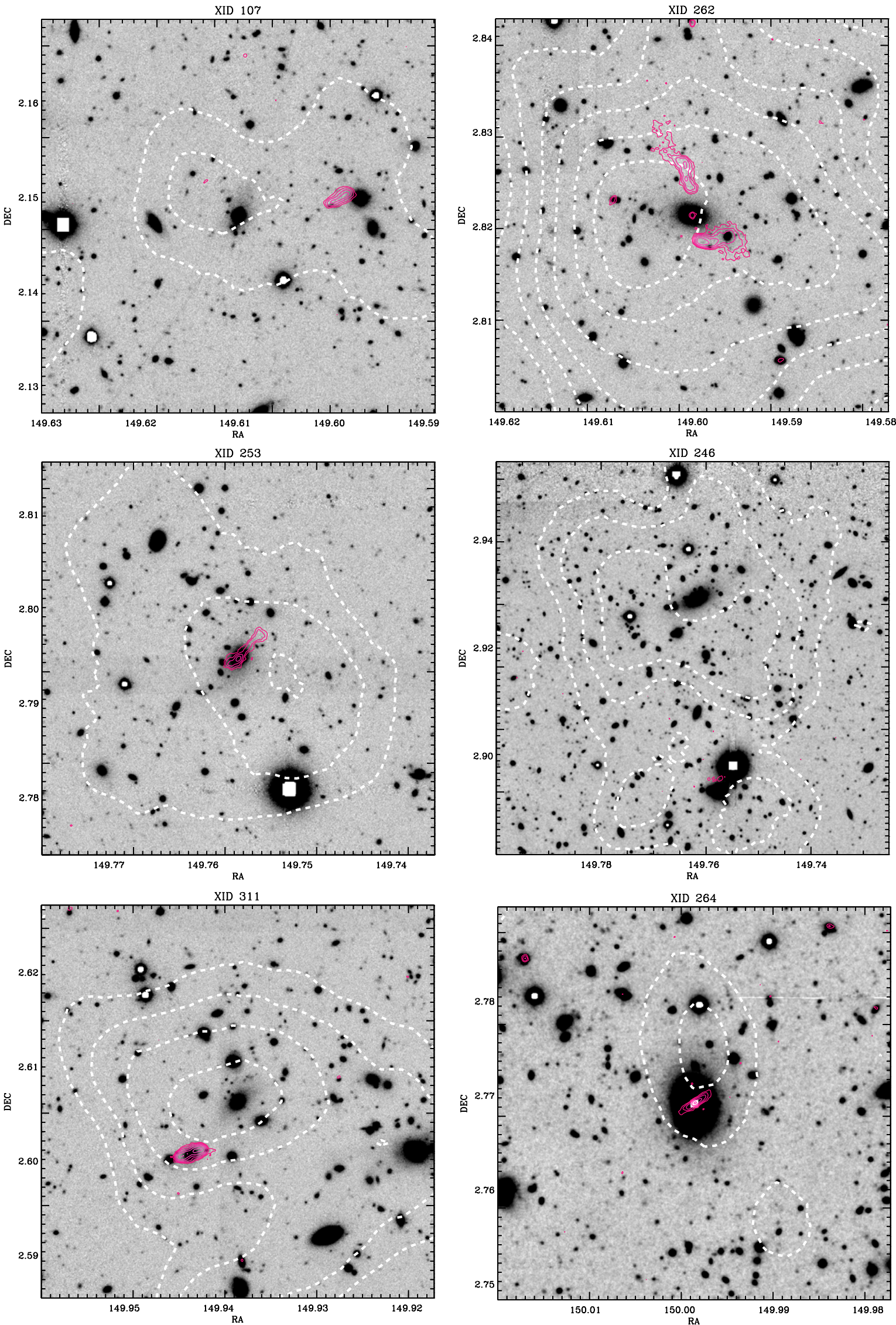

Figure 4. Contours maps of the radio $20 \mathrm{~cm}$ emission (solid lines) superimposed to the Subaru $z p$ band image for each of the groups listed in Table 1. (A color version of this figure is available in the online journal.) 

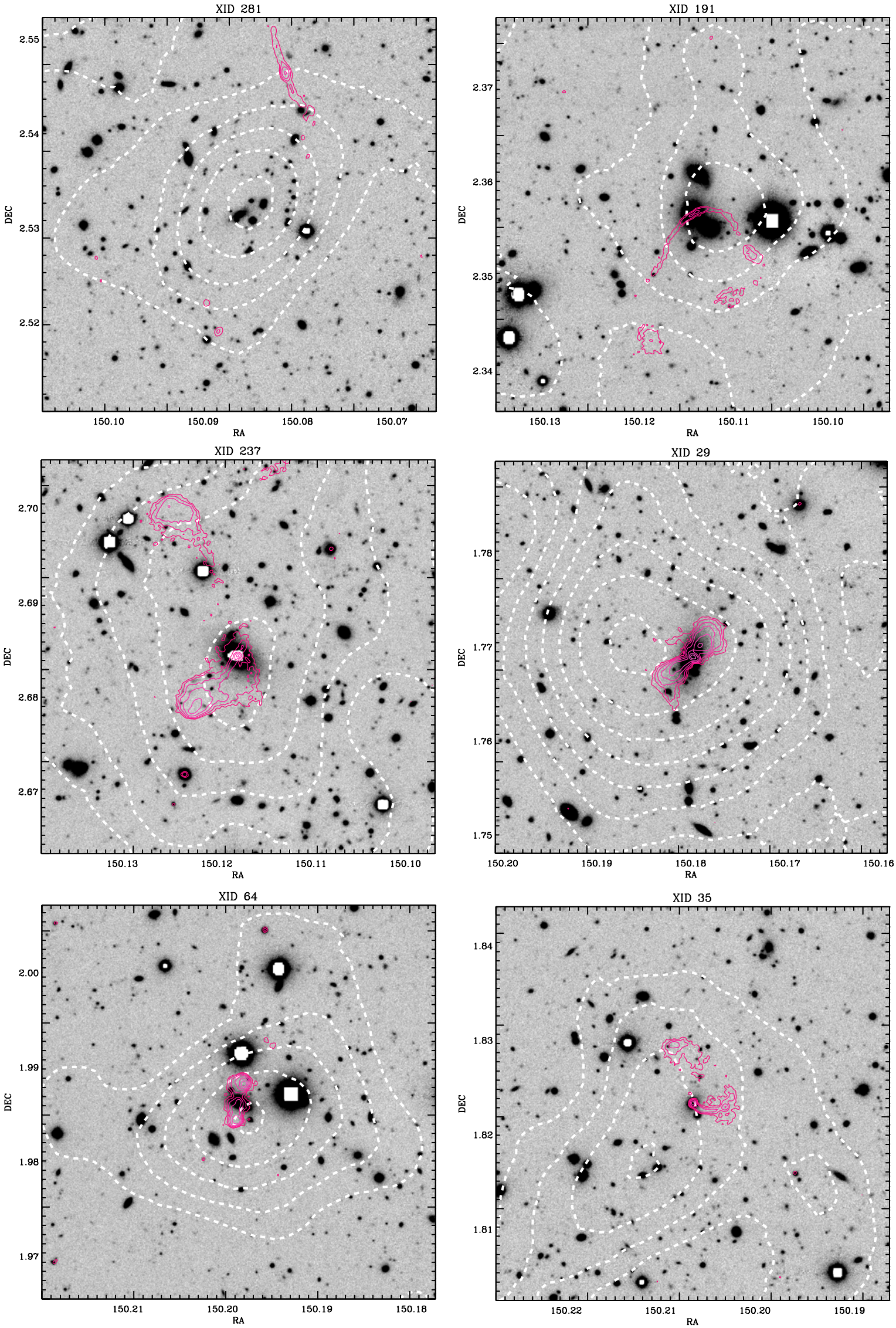

Figure 4. (Continued) 

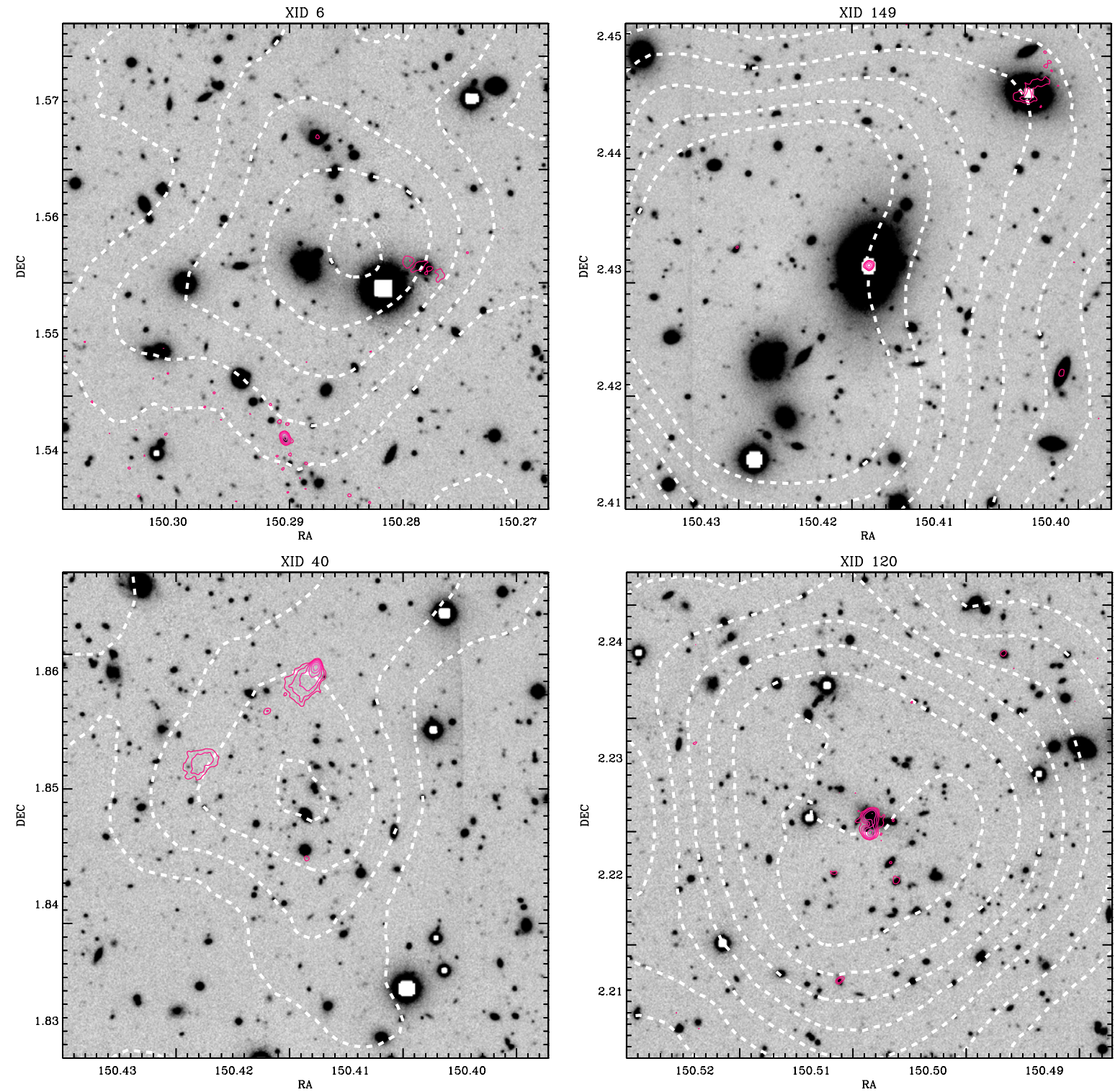

Figure 4. (Continued)

contours correspond to $[3,6,9,12,15,18,21,24] \sigma \mathrm{X}$-ray flux significance.

\section{REFERENCES}

Arnaud, M., \& Evrard, A. E. 1999, MNRAS, 305, 631

Balogh, M. L., McCarthy, I. G., Bower, R. G., \& Eke, V. R. 2008, MNRAS, 385, 1003

Baum, S. A., Heckman, T. M., \& van Breugel, W. 1992, ApJ, 389, 208

Bîrzan, L., McNamara, B. R., Nulsen, P. E. J., Carilli, C. L., \& Wise, M. W. 2008, ApJ, 686, 859

Bîrzan, L., Rafferty, D. A., McNamara, B. R., Wise, M. W., \& Nulsen, P. E. J. 2004, ApJ, 607, 800

Bower, R. G., Benson, A. J., Malbon, R., Helly, J. C., Frenk, C. S., Baugh, C. M., Cole, S., \& Lacey, C. G. 2006, MNRAS, 370, 645

Bower, R. G., McCarthy, I. G., \& Benson, A. J. 2008, MNRAS, 390, 1399

Chen, Y., Reiprich, T. H., Böhringer, H., Ikebe, Y., \& Zhang, Y.-Y. 2007, A\&A, 466, 805

Churazov, E., Sunyaev, R., Forman, W., \& Böhringer, H. 2002, MNRAS, 332, 729

Condon, J. J., Cotton, W. D., Greisen, E. W., Yin, Q. F., Perley, R. A., Taylor, G. B., \& Broderick, J. J. 1998, AJ, 115, 1693

Croton, D. J., et al. 2006, MNRAS, 365, 11

De Young, D. S. 2010, ApJ, 710, 743
Donahue, M., Voit, G. M., O’Dea, C. P., Baum, S. A., \& Sparks, W. B. 2005, ApJ, 630, L13

Drory, N., Bender, R., Feulner, G., Hopp, U., Maraston, C., Snigula, J., \& Hill, G. J. 2004, ApJ, 608, 742

Dunkley, J., et al. 2009, ApJS, 180, 306

Evrard, A. E., Metzler, C. A., \& Navarro, J. F. 1996, ApJ, 469, 494

Fabian, A. C., Arnaud, K. A., Bautz, M. W., \& Tawara, Y. 1994, ApJ, 436, L63

Feretti, L., \& Giovannini, G. 2008, in A Pan-Chromatic View of Clusters of Galaxies and the Large-Scale Structure, ed. M. Plionis, O. Lóópez Cruz, \& D. Hughes (Dordrecht: Springer), 143

Finoguenov, A., Ruszkowski, M., Jones, C., Brüggen, M., Vikhlinin, A., \& Mandel, E. 2008, ApJ, 686, 911

Giodini, S., et al. 2009, ApJ, 703, 982

Gitti, M., McNamara, B. R., Nulsen, P. E. J., \& Wise, M. W. 2007, ApJ, 660, 1118

Hayashi, E., Navarro, J. F., \& Springel, V. 2007, MNRAS, 377, 50

Heinz, S., Reynolds, C. S., \& Begelman, M. C. 1998, ApJ, 501, 126

Ilbert, O., et al. 2009, ApJ, 690, 1236

Kaastra, J. S., et al. 2004, A\&A, 413, 415

Kay, S. T. 2004, MNRAS, 347, L13

Leauthaud, A., et al. 2010, ApJ, 709, 97

Lin, Y.-T., Mohr, J. J., \& Stanford, S. A. 2003, ApJ, 591, 749

Lloyd-Davies, E. J., Ponman, T. J., \& Cannon, D. B. 2000, MNRAS, 315, 689

Macciò, A. V., Dutton, A. A., van den Bosch, F. C., Moore, B., Potter, D., \& Stadel, J. 2007, MNRAS, 378, 55 
Markevitch, M. 1998, ApJ, 504, 27

McCarthy, I. G., Bower, R. G., \& Balogh, M. L. 2007, MNRAS, 377, 1457

McNamara, B. R., \& Nulsen, P. E. J. 2007, ARA\&A, 45, 117

Merloni, A., \& Heinz, S. 2007, MNRAS, 381, 589

Myers, S. T., \& Spangler, S. R. 1985, ApJ, 291, 52

Navarro, J. F., Frenk, C. S., \& White, S. D. M. 1996, ApJ, 462, 563

Nipoti, C., \& Binney, J. 2005, MNRAS, 361, 428

Peres, C. B., Fabian, A. C., Edge, A. C., Allen, S. W., Johnstone, R. M., \& White, D. A. 1998, MNRAS, 298, 416

Peterson, J. R., Kahn, S. M., Paerels, F. B. S., Kaastra, J. S., Tamura, T., Bleeker, J. A. M., Ferrigno, C., \& Jernigan, J. G. 2003, ApJ, 590, 207

Peterson, J. R., \& Fabian, A. C. 2006, Phys. Rep., 427, 1

Ponman, T. J., Sanderson, A. J. R., \& Finoguenov, A. 2003, MNRAS, 343, 331

Pratt, G. W., \& Arnaud, M. 2003, A\&A, 408, 1

Pratt, G. W., Croston, J. H., Arnaud, M., \& Böhringer, H. 2009, A\&A, 498, 361

Pratt, G. W., et al. 2009, A\&A, in press (arXiv:0909.3776)

Puchwein, E., Sijacki, D., \& Springel, V. 2008, ApJ, 687, L53
Reiprich, T. H., \& Boehringer, H. 2002, ApJ, 567, 716

Renzini, A. 2006, ARA\&A, 44, 141

Sanderson, A. J. R., \& Ponman, T. J. 2003, MNRAS, 345, 124

Salvato, M., et al. 2009, ApJ, 690, 1250

Scoville, N., et al. 2007, ApJS, 172,

Schinnerer, E., et al. 2007, ApJS, 172, 46

Sijacki, D., \& Springel, V. 2006, MNRAS, 366, 397

Short, C. J., \& Thomas, P. A. 2009, ApJ, 704, 915

Skrutskie, M. F., et al. 2006, AJ, 131, 1163

Smolčić, V., et al. 2008, ApJS, 177, 14

Smolčić, V., et al. 2009, ApJ, 696, 24

Sun, M., Voit, G. M., Donahue, M., Jones, C., Forman, W., \& Vikhlinin, A. 2009, ApJ, 693, 1142

Voit, G. M., \& Donahue, M. 2005, ApJ, 634, 955

Voigt, L. M., \& Fabian, A. C. 2004, MNRAS, 347, 1130

Voit, L. M. 2005, Rev. Mod. Phys., 77, 207

Wright, A., \& Otrupcek, R. 1990, PKS Catalog, 0 